\section{SP0144 THE IMPORTANCE OF MAINTAINING CONTROL}

K.E.N. Clark. University College London, London, UK

The clinical impact of lower gastrointestinal tract involvement in systemic sclerosis will be illustrated by case presentations. This will describe problems including pseudo-obstruction, severe malnutrition, and electrolyte imbalance and anorectal disease. The interplay with comorbidities such as cardiac scleroderma will also be described. Potential therapeutic strategies for these different complications will be introduced through these cases.

Disclosure of Interest: None declared

DOI: 10.1136/annrheumdis-2018-eular.7672

\section{SP0145 LIGHT AT THE LOWER GASTROINTESTINAL TRACT IN SYSTEMIC SCLEROSIS}

C.P. Denton. Centre for Rheumatology, Royal Free Hospital and UCL Medical School, London, UK

Gastrointestinal tract manifestations of systemic sclerosis are common and represent a high burden of the disease. Whilst some aspects can be treated the lower bowel involvement is especially challenging. The midgut is affected with dysmotility and functional impairment including the consequences of impaired absorption and exocrine pancreatic insufficiency. Midgut hypomotility can lead to stagnation of bowel contents and small intestinal bacterial overgrowth that contributes to bloating, diarrhoea and malnutrition. Colonic involvement contributes to chronic constipation and anorectal disease is a major non-lethal burden leading to incontinence. This has enormous impact on quality of life. The end result is a constellation of symptoms and clinical problems that require integrated management to ensure appropriate investigation and treatment. Strategies that can be helpful include broad spectrum antibiotics to address small intestinal bacterial overgrowth and prokinetics to address issues of pseudo-obstruction. The latter is best managed conservatively. Occasionally patients develop nutritional failure that requires parental nutrition. This can be successfully delivered as part of a home care programme and is generally parenteral supplementation, for example with overnight feeds, rather than total parenteral nutrition. Constipation and diarrhoea require opposite strategies for treatment and often a high degree of patient self-management. Recent trials of techniques to improve anorectal incontinence have been promising for strategies such as posterior tibial nerve stimulations and this may represent useful option in some cases.

Disclosure of Interest: C. Denton Grant/research support from: Inventiva, CSL Behring, GSK, Bayer, Consultant for: GSK, Actelion, Inventiva, Roche, Bayer, Boehringer Ingelheim, EMD Serono, Sanofi Aventis

DOI: 10.1136/annrheumdis-2018-eular.7621

SATURDAY, 16 JUNE 2018

\section{RA: is it all in your head?}

\section{SP0146 THE LINK BETWEEN INFLAMMATION AND DEPRESSION}

N. Harrison. Neuroscience, Brighton and Sussex Medical School, Brighton, UK

Inflammation is increasingly implicated in the etiology of depression. Patients with idiopathic depression have been shown to express increased circulating proinflammatory cytokines and $30 \%$ of patients receiving chronic Interferon-alpha for the treatment of Hepatitis-C develop major depression. Recently, anti-inflammatory agents, including 'biologics' developed for rheumatoid arthritis, have shown potential efficacy for the treatment of idiopathic depression. However, the utility of these medications is highly variable emphasising the importance of identifying biomarkers that may predict treatment response. Interestingly, neuroimaging studies have recently demonstrated that increases in inflammation can rapidly alter brain function and microstructural integrity. Further, these acute changes in brain structure/function can additionally predict the later development of depressive response during sustained inflammatory challenge. For example, in patients with Hepatitis-C given Interferon-alpha over a prolonged period, acute changes in ventral striatal microstructure (observed within 4 hours of the first injection of Interferon) predict the magnitude of fatigue/motivational impairment experienced 4 weeks later while acute changes in amygdala processing of emotionally valenced stimuli predicts the magnitude of depressive symptoms. Conversely, inhibitors of Tumour Necrosis Factor (TNF) reduce amygdala reactivity to emotional stimuli, which correlate with improvement in mood. Together, these studies highlight the importance of inflammatory processes in depression and support ongoing trials of anti-inflammatory agents in this common and functionally impairing disorder.

Disclosure of Interest: N. Harrison Grant/research support from: Janssen, Consultant for: GSK

DOI: 10.1136/annrheumdis-2018-eular.7813

\section{SATURDAY, 16 JUNE 2018}

\section{Gut bacteria: the boss of the immune system}

\section{SP0147 HOW THE GUT MICROBIOTA AND ITS METABOLITES IMPACT ON BONE HOMEOSTASIS}

M.M. Zaiss. Department of Internal Medicine 3 - Rheumatology and Immunology, Friedrich-Alexander-University Erlangen-Nürnberg (FAU), Erlangen, Germany

Microbial metabolites are known to modulate immune responses of the host. The main metabolites derived from microbial fermentation of dietary fibres in the intes tine, short-chain fatty acids (SCFA), affect local and systemic immune functions. Here we show that SCFA are regulators of osteoclast metabolism and bone mass in vivo. Treatment of mice with SCFA as well as feeding with a high-fibre diet significantly increases bone mass and prevents postmenopausal and inflammation induced bone loss. The protective effects of SCFA on bone mass are associated with inhibition of osteoclast differentiation and bone resorption in vitro and in vivo while bone formation is not affected. Mechanistically, propionate (C3) and butyrate $(\mathrm{C} 4)$ induce metabolic reprogramming of osteoclasts resulting in enhanced glycolysis at the expense of oxidative phosphorylation, thereby downregulating essential osteoclast genes such as TRAF6 and NFATc1. In summary, these data identify SCFA as potent regulators of osteoclast metabolism and bone homeostasis.

Disclosure of Interest: None declared

DOI: 10.1136/annrheumdis-2018-eular.7900

\section{SATURDAY, 16 JUNE 2018}

\section{Multi-disciplinary management of complex persistent pain}

SP0148 PAIN: THE ROLE OF PSYCHOLOGY

L. Knudsen. Spinal Cord Injury Centre of Western Denmark, Viborg, Denmark

Pain is a multidimensional experience, and chronic ongoing pain may have wide ranging social and psychological implications. A biopsychosocial model of understanding is generally considered to be the best pain model, and it is well-established that interdisciplinary pain management is the most effective treatment approach. Psychological management plays an important role in such treatment. This lecture will cover the link between psychological factors and pain as well as psychological pain management.

Disclosure of Interest: None declared

DOI: 10.1136/annrheumdis-2018-eular.7799

\section{SP0149 THE PATIENT'S PERSPECTIVE OF LIVING WITH COMPLEX REGIONAL PAIN SYNDROME}

R. Connett. Select, Royal Devon and Exeter Hospital, Exeter, Devon, UK, Exeter, UK

There is wide acknowledgement in medical literature that CRPS is a very painful condition that can be severely debilitating and adversely affect quality of life. In an attempt to assist the many highly caring, gifted and dedicated clinicians who want to understand what this actually means from the perspective of those who live with the condition relentlessly day by day, I shall attempt to bring such a statement to life. I shall speak mainly from my own journey of learning to live with CRPS since 2001 , but shall also draw on the experiences fellow sufferers have shared with me, and humbly contribute some key messages for the clinicians who come into contact with patients pre or post diagnosis.

Disclosure of Interest: None declared

DOI: 10.1136/annrheumdis-2018-eular.7836 